\title{
Zum Stand der Verordnungsverfahren des Bundesministeriums für Umwelt, Naturschutz und Reaktorsicherheit im Bereich des Grundwasser- und Bodenschutzes und der Verwertung von mineralischen Ersatzbaustoffen - Notwendigkeit der geplanten Mantelverordnung
}

\author{
Bernd Susset • Peter Grathwohl
}

Online veröffentlicht: 20.10 .2011

(C) Springer-Verlag 2011

Grundwasser unterliegt zunehmend einer konkurrierenden Nutzung. Dies betrifft nicht nur die Entnahme von Wasser für verschiedene Zwecke oder die geothermische Nutzung sondern auch die Landnutzung und mehr und mehr auch bauliche Eingriffe in den Untergrund. Zur Schonung der Ressourcen und damit ganz im Sinne des Kreislaufwirtschafts- und Abfallgesetzes (derzeit liegt ein Referentenentwurf für ein neues Abfallgesetz - das Kreislaufwirtschaftsgesetz - vor) werden zunehmend mineralische Recyclingmaterialien (z. B. aufbereiteter Bauschutt und industrielle Abfälle) in der Landschaftsgestaltung, im Straßen- und Wegebau oder direkt als Bauprodukt eingesetzt. Anforderungen des Grundwasserschutzes sollten deshalb beim Umgang mit Boden, Bauprodukten und mineralischen Ersatzbaustoffen sowie Düngemitteln etc. berücksichtigt werden. Es liegt im allgemeinen Interesse, dass die verschiedenen Verordnungen harmonisiert werden und Verordnungsnovellen oder Neuverordnungen wie die Novelle der BundesBodenschutzverordnung, die Bauproduktenrichtlinie, die geplante Ersatzbaustoffverordnung und die Düngemittelverordnung den Anforderungen des Grundwasserschutzes gerecht werden. Deshalb plant der Gesetzgeber neben der Grundwasserverordnung eine Mantelverordnung auf den Weg zu bringen, die den Grundwasserschutz verschiedener Bereiche integriert.

Die neue Grundwasserverordnung (GrwV vom 9. November 2010) setzt die EU-Bestimmungen der Wasser-

Dr. B. Susset $(\bowtie)$ · Prof. Dr. P. Grathwohl

Zentrum für Angewandte Geowissenschaften,

Eberhard-Karls-Universität Tübingen,

Sigwartstr. 10, 72076 Tübingen, Deutschland

E-Mail: bernd.susset@ifg.uni-tuebingen.de

Prof. Dr. P. Grathwohl

E-Mail: grathwohl@uni-tuebingen.de rahmenrichtlinie 1:1 in nationales Recht um. Es wurden Schwellenwerte für die 10 EU-Pflichtparameter festgelegt (siehe Beitrag von Herrn Keppner in Heft 3/2011, S. 145153) - eine Konkretisierung des sogenannten Besorgnisgrundsatzes nach $\$ 48$ des Wasserhaushaltsgesetzes, also die Festlegung an welchem Ort und über welchen Zeitraum die Schwellenwerte gelten, fehlt aber noch weitestgehend (inwiefern sind kurzfristige und kleinräumige Überschreitungen tolerierbar?). Aufgrund der kontroversen Diskussion und angesichts der durch das drohende Vertragsverletzungsverfahren der EU gegen die Bundesrepublik Deutschland wegen der verspäteten Festlegung der EU-Pflichtparameter drängenden Zeit wurden solche Konkretisierungen sowie die Verrechtlichung eines erweiterten Katalogs von Stoffgrenzwerten zunächst zurückgestellt.

Am 06.01.2011 hat das BMU den lange erwarteten ersten Entwurf der Mantelverordnung (MantelV) zur Festlegung von Anforderungen an das Einbringen und Einleiten von Stoffen in das Grundwasser (Artikel 1 der MantelV: Novelle der Grundwasserverordnung, GrundwasserV), an den Einbau von mineralischen Ersatzbaustoffen (Artikel 2 der MantelV: Neuverordnung der Ersatzbaustoffverordnung, ErsatzbaustoffV) und für die Verwendung von Boden und bodenähnlichem Material (Artikel 3 der MantelV: Novelle der Bundes-Bodenschutzverordnung, BBodSchV) vorgelegt. In Artikel 1 ist geplant, als Ergänzung der GrundwasserV, neben den 10 EU-Schwellenwerten für 46 weitere Stoffe sogenannte Prüfwerte (entsprechend der jeweiligen Geringfügigkeitsschwellenwerte der Länderarbeitsgemeinschaft Wasser) für das Grundwasser festzulegen und den Besorgnisgrundsatz dahingehend zu konkretisieren, dass eine nachteilige Veränderung des Grundwassers dann nicht zu erwarten ist, wenn die Prüfwerte im Grundwasser unmittelbar nach Eintritt der Stoffe ins Grundwasser eingehalten werden und die zu erwartenden Schadstoffmengen gering 
sind. Bei Überschreitung kann im Einzelfall geprüft werden, ob dennoch eine Grundwasserbenutzung erlaubt werden kann (deshalb die Bezeichnung Prüfwerte). Bei direkten Schadstoffeinträgen durch Bauprodukte in das Grundwasser (z. B. Betonpfeiler im Grundwasser) erfolgt eine Betrachtung der über ein räumlich begrenztes Volumen und einen kurzen Zeitraum gemittelten Konzentrationen (diese werden zum Prüfwertvergleich herangezogen, die Konkretisierung erfolgt im Rahmen bauaufsichtlicher Zulassungen des Deutschen Instituts für Bautechnik). Lediglich die zuletzt genannten direkten Schadstoffeinträge in das Grundwasser stellen eine ,echte“ Benutzung des Grundwassers im Sinne des Wasserhaushaltsgesetzes dar. Die Verwendung von Bodenmaterialien und mineralischen Ersatzbaustoffen oberhalb des Grundwassers sind „unechte“ Benutzungen und werden durch die GrundwasserV nicht direkt geregelt. Die Prüfwerte der GrundwasserV stellen jedoch - fachlich konsequent - Zielgrößen für die Gefahrenbeurteilung dar und wirken sich somit auch auf die materiellen Anforderungen an Boden und bodenähnliche Materialien bzw. mineralische Ersatzbaustoffe in der Novelle der BBodSchV und in der ErsatzbaustoffV aus. In der Novelle der BBodSchV (Artikel 3 der MantelV) wird für die erlaubnisfreie (d. h. ohne wasserrechtliche Genehmigung) Verwendung von Boden und bodenähnlichen Materialien eine direkte Einhaltung u. a. der in der GrundwasserV (Artikel 1) geregelten Prüfwerte oder von methodenspezifischen Hintergrundwerten in genormten Laboreluaten - im Gegensatz zur ErsatzbaustoffV ohne Berücksichtigung der Rückhaltung im Untergrund - gefordert. Insbesondere wird hier durch die Ergänzungen in $\$ 12$ der Novelle BBodSchV (siehe Artikel 3 der MantelV) die ,Verfüllung von Abgrabungen“ erstmalig bundeseinheitlich geregelt. In der ErsatzbaustoffV (Artikel 2 der MantelV) gelten sogenannte Materialwerte im Eluat, die je nach Untergrundkonstellation ggf. die Rückhaltung in Böden berücksichtigen (vorgezogene Sickerwasserprognosen für standardisierte Einbauweisen und Standardszenarien).

Die Geowissenschaften haben einen maßgeblichen Beitrag zum Fachkonzept insbesondere der geplanten ErsatzbaustoffV geleistet. Das Konzept beruht insbesondere auf den Ergebnissen des Forschungsverbundes Sickerwasserprognose des BMBF, an dem auch viele Hydrogeologen aus Forschungseinrichtungen und der Praxis beteiligt waren. Für gängige Bauweisen (z. B. Straßendämme, Tragschichten, ungebundene Deckschichten) wurden die zu erwartenden Stoffausträge und deren zeitlicher Verlauf be- stimmt (Bestimmung des Quellterms). Der Quellterm fungiert hier als Eingangsgröße für vorgezogene Transportprognosen von Standardszenarien (bestimmte Untergrundkonstellationen, Transportterm), um die Rückhaltung in Böden „auf der sicheren Seite“ abzuschätzen. Im Ergebnis resultieren für die ErsatzbaustoffV einzuhaltende, so genannte Materialwerte in einem genormten Säuleneluat und damit verbunden zulässige Einbaukonfigurationen von mineralischen Ersatzbaustoffen. Für diese entfällt dann wiederum ein wasserrechtliches Einzelfallgenehmigungsverfahren. Die nach den Grundsätzen des vorsorgenden Boden- und Grundwasserschutzes abgeleiteten Grenzwerte für mineralische Ersatzbaustoffe und zulässigen Bauweisen werden auch nach Vorlage des zweiten Arbeitsentwurfs der ErsatzbaustoffV in Artikel 2 der MantelV von Januar dieses Jahres kontrovers diskutiert.

Die MantelV sieht eine integrierte Betrachtung von Grundwasserschutz, Bodenschutz und mineralischen Ersatzbaustoffen vor. Damit wurde den Forderungen der Akteure sowohl aus der Forschung als auch der Industrie nach einem ganzheitlichen Konzept Rechnung getragen. Man erhofft sich hierdurch deutliche Erleichterungen für den Vollzug und die Wirtschaft. Da solche Rechtsregelungen wesentlich wirtschaftliches Handeln bestimmen, verursachen sie je nach Ausprägung natürlich intensive Diskussionen zwischen den verschiedenen Interessensgruppen. Unbestritten ist, dass Regelungen zum Schutz der öffentlichen Güter unabdingbar sind und deren Ableitung nicht bis zur Klärung sämtlicher evtl. bestehender Unsicherheiten aufgeschoben werden kann. Deshalb müssen Regelwerke wie zum Beispiel die geplante ErsatzbaustoffV zur bundeseinheitlichen Regelung der Verwertung von mineralischen Ersatzbaustoffen (diese findet bereits jetzt verbreitet Zuspruch) nun dringend auf den Weg gebracht werden. Darüber hinaus müssen die bei der Erarbeitung von fachtechnischen Konzepten für Verordnungsvorhaben aufgetretenen neuen wissenschaftlichen Fragestellungen (z. B. die Berücksichtigung des ggf. repräsentativ für ein Material belegbaren Abklingverhaltens von Schwermetallen im Sickerwasser zur schichtspezifischen Bewertung des Gefährdungspotentials von mineralischen Ersatzbaustoffen in dünnschichtigen Bauweisen wie Sportplatzbelägen oder gegenüber Lärmschutzwällen geringer mächtigen Tragschichten) auch nach Abschluss der Verordnungsverfahren wissenschaftlich bearbeitet und weiter berücksichtigt werden. 